\title{
CONTROLLING HYPER CHAOS WITH FEEDBACK OF DYNAMICAL VARIABLES
}

\author{
XIAO-SHU LUO \\ Department of Modern Physics, University of Science and Technology of China, \\ Hefei 230026, China; and Department of Physics and Electronic Science, \\ Guangxi Normal University, Guilin, 541004, China \\ BING-HONG WANG* \\ Department of Modern Physics and Nonlinear Science Center, \\ University of Science and Technology of China, Hefei 230026, China \\ bhwang@ustc.edu.cn
}

Received 8 August 2002

\begin{abstract}
We propose a method for controlling chaos and hyper-chaos by applying continuous proportional feedback to the system variables and their derivatives. The method has been applied successfully in six-order coupled Chua's hyper-chaotic circuit system. The theoretical analysis and numerical simulation results show that unstable fixed points embedded in hyper-chaotic attractors can be stabilized and Hopf bifurcation can be observed for the controlled system.
\end{abstract}

Keywords: Chaos controlling; continuous proportional feedback of the system variables; Chua's chaotic circuit.

In recent ten years, chaos controlling has become one of the frontier topics of great interests in nonlinear science since the pioneering work done by Ott-Grebogi-York (OGY). ${ }^{1}$ Due to its great potential of application, many different methods for chaos controlling in various chaotic systems have been proposed. ${ }^{2-8}$ Two kinds among them are special significant from the point of view for controlling strategy. First, some small perturbations can be applied on system's parameters in order to stabilize unstable periodic orbits (UPOs) embedded in the chaotic attractor. ${ }^{1-3}$ Second, chaos can be controlled based on feedback of system variables, for example, timedelay feedback, ${ }^{4}$ a direct proportional impulse feedback. ${ }^{5}$ and a digital filter ${ }^{6}$ of system variables. However, most available methods of chaos controlling based on feedback of system variables are not suitable for controlling hyper-chaos in highdimensional nonlinear dynamical systems with more than one positive Lyapunov exponents. ${ }^{7}$ In this paper, we propose a new method based on feedback of system 
variables which is not only suitable for controlling chaos but also suitable for hyperchaos. The new method has been applied to six-order coupled Chua's hyper-chaotic circuit system. The theoretical analysis and numerical simulation results show that unstable fixed points embedded in hyper-chaotic attractors can be stabilized much effectively and Hopf bifurcation can be observed for the controlled system.

Let us consider a $n$-dimensional autonomous nonlinear dynamical system described by the differential equations

$$
\dot{x}=f(x, \mu)
$$

where the state vector $x \in R^{n}$ is a $n$-dimensional vector with components $x_{1}, x_{2}, \ldots, x_{n}, f$ is a $n$-dimensional smooth vector field with components $f_{1}, f_{2}, \ldots, f_{n}$, and $\mu$ represents a set of system parameters. The system described by Eq. (1) is chaotic for parameter region $\mu \in M$ corresponding to chaos state. Our method for controlling chaos and hyper-chaos can be described as follows.

Suppose dynamical variables $x_{i}$ and $\dot{x_{i}}$ for a special subscript $i$ can be measured. Make a new system variable $x_{i}^{\prime}$ in Eq. (1) as

$$
x_{i}^{\prime}=k_{1} x_{i}+k_{2} \dot{x_{i}}
$$

where $k_{1}$ and $k_{2}$ are two adjustable parameters, then feeding $x_{i}^{\prime}$ into the $j$-th subsystem of Eq. (1) will lead to the controlled system

$$
\left\{\begin{array}{l}
\dot{x}_{j}=f_{j}\left(x_{1}, \ldots, x_{i-1}, x_{i}^{\prime}, x_{i+1}, \ldots, x_{n}\right) \\
\dot{x}_{l}=f_{l}\left(x_{1}, \ldots, x_{i-1}, x_{i}, x_{i+1}, \ldots, x_{n}\right) l=1, \ldots j-1, j+1, \ldots, n(l \neq j) . \\
x_{i}^{\prime}=k_{1} x_{i}+k_{2} \dot{x}_{i}
\end{array}\right.
$$

However, for controlling hyper-chaos, it is imperative that two new variables $x_{i_{1}}^{\prime}$ and $x_{i_{2}}^{\prime}$ should be fed into at least two subsystems, for example, the $j_{1}$-th subsystem and the $j_{2}$-th subsystem of Eq. (1). Then the controlled system for hyper-chaos controlling takes the form

$$
\left\{\begin{array}{l}
\dot{x}_{j_{1}}=f_{j_{1}}\left(x_{1}, \ldots, x_{i_{1}-1}, x_{i_{1}}^{\prime}, x_{i_{1}+1}, \ldots, x_{i_{2}-1}, x_{i_{2}}^{\prime}, x_{i_{2}+1}, \ldots x_{n}\right) \\
\dot{x}_{j_{2}}=f_{j_{2}}\left(x_{1}, \ldots, x_{i_{1}-1}, x_{i_{1}}^{\prime}, x_{i_{1}+1}, \ldots, x_{i_{2}-1}, x_{i_{2}}^{\prime}, x_{i_{2}+1}, \ldots x_{n}\right) \\
\dot{x}_{l}=f_{l}\left(x_{1}, \ldots, x_{i_{1}}, \ldots, x_{i_{2}}, \ldots, x_{n}\right) \\
x_{i_{1}}^{\prime}=k_{1} x_{i_{1}}+k_{2} \dot{x}_{i_{1}} \\
x_{i_{2}}^{\prime}=k_{3} x_{i_{2}}+k_{4} \dot{x}_{i_{2}}
\end{array}\left(l \neq j_{1}, j_{2}\right) .\right.
$$

The dimensionless equations of the six-th order coupled Chua's circuit are given by

$$
\left\{\begin{array}{l}
\dot{x}_{1}=\alpha \cdot\left(x_{2}-x_{1}-f\left(x_{1}\right)\right) \\
\dot{x}_{2}=x_{1}-x_{2}+x_{3}+\gamma\left(x_{5}-x_{2}\right) \\
\dot{x}_{3}=-\beta x_{2} \\
\dot{x}_{4}=\alpha \cdot\left(x_{5}-x_{4}-f\left(x_{4}\right)\right) \\
\dot{x}_{5}=x_{4}-x_{5}+x_{6} \\
\dot{x}_{6}=-\beta \cdot x_{5}
\end{array}\right.
$$




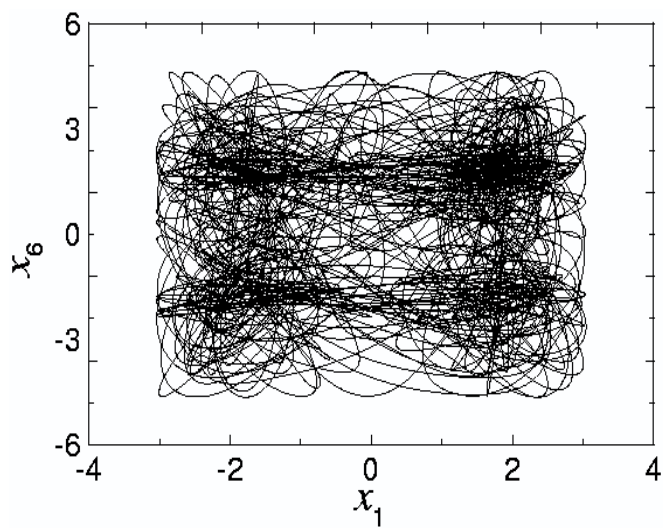

Fig. 1. Hyper-chaotic attractor of six order coupled Chua's circuit for the parameters $\alpha=10.0$, $\beta=14.87, \gamma=0.02$, and $a=-1.27, b=-0.68$.

where the functional form $f(x)$ is:

$$
f(x)=b x+\frac{(a-b)}{2}(|x+1|-|x-1|)
$$

The six-th order coupled Chua's circuit can show hyper-chaos in Fig. 1 for the parameters $\alpha=10.0, \beta=14.87, \gamma=0.02$, and $a=-1.27, b=-0.68 .^{9}$ It turns out that feedback of only a single variable into one subsystem is not enough for hyper-chaos controlling. At least two feedback variables have to be fed into more than two subsystems is necessary for controlling hyper-chaos in high dimensional nonlinear system. Here, we select $x_{1}$ and $x_{4}$ as feedback variables, and feed $x_{1}^{\prime}$ and $x_{4}^{\prime}$ into the $2^{\text {nd }}$ and the $5^{\text {th }}$ subsystem of Eq. (5). Then the controlled system for the $6^{\text {th }}$ order Chua's circuit can be written as

$$
\left\{\begin{array}{l}
\dot{x}_{1}=\alpha \cdot\left(x_{2}-x_{1}-f\left(x_{1}\right)\right) \\
\dot{x}_{2}=x_{1}^{\prime}-x_{2}+x_{3}+\gamma\left(x_{5}-x_{2}\right) \\
\dot{x}_{3}=-\beta x_{2} \\
\dot{x}_{4}=\alpha \cdot\left(x_{5}-x_{4}-f\left(x_{4}\right)\right) \\
\dot{x}_{5}=x_{4}^{\prime}-x_{5}+x_{6} \\
\dot{x}_{6}=-\beta x_{5} \\
x_{1}^{\prime}=x_{1}+k_{2} \dot{x}_{1} \\
x_{4}^{\prime}=x_{4}+k_{3} \dot{x}_{4}
\end{array}\right.
$$

The Jacobian matrix $J_{2}$ of the controlled hyper-chaotic system (7) at its fixed point is: 


$$
J_{2}=\left[\begin{array}{cccccr}
-\alpha-\alpha \cdot f^{\prime}\left(x_{1 e}\right) & \alpha & 0 & 0 & 0 & 0 \\
-k_{2} \alpha-k_{2} \alpha \cdot f^{\prime}\left(x_{1 e}\right)+1 & k_{2} \alpha-1-\gamma & 1 & 0 & \gamma & 0 \\
0 & -\beta & 0 & 0 & 0 & 0 \\
0 & 0 & 0 & -\alpha-\alpha \cdot f^{\prime}\left(x_{4 e}\right) & \alpha & 0 \\
0 & 0 & 0 & -k_{3} \alpha-k_{3} \alpha \cdot f^{\prime}\left(x_{4 e}\right)+1 & k_{3} \alpha-1 & 1 \\
0 & 0 & 0 & 0 & -\beta & 0
\end{array}\right] .
$$

Hence the eigen-value equation of the fixed points can be derived as $\left|\lambda I-J_{2}\right|=0$. The value range of $k_{2}$ and $k_{3}$ corresponding to stable fixed points:

$$
\begin{aligned}
& A_{1}(-1.84375,0,1.84375,-1.84375,0,1.84375) ; \\
& A_{2}(-1.84375,0,1.84375,1.84375,0,-1.84375) ; \\
& A_{3}(1.84375,0,-1.84375,-1.84375,0,1.84375) ; \\
& A_{4}(1.84375,0,-1.84375,1.84375,0,-1.84375)
\end{aligned}
$$

can be determined respectively as $-20.0 \leq k_{2} \leq-0.2$, and $-20.0 \leq k_{3} \leq-0.2$. The Hopf bifurcation can also happen at the critical point of the parameters: $k_{2}=$ $-0.2, k_{3}=-0.12$. A series of period-doubling bifurcations can be observed when the control parameters $k_{2}$ and $k_{3}$ increase from their critical values. By using Poincaré map in the surface section $x_{2}=x_{5}=0$, a typical bifurcation diagram can be obtained as shown in Fig. 2. Figure 3(a) shows the controlling process approaching to fixed point $A_{1}$ for $k_{2}=-0.2$ and $k_{3}=-0.3$. The stabilized period-2 limit cycle embedded in hyper-chaos of the controlled sixth order coupled Chua's circuit with $k_{2}=-0.2, k_{3}=-0.12$ is shown in Fig. $3(\mathrm{~b})$.

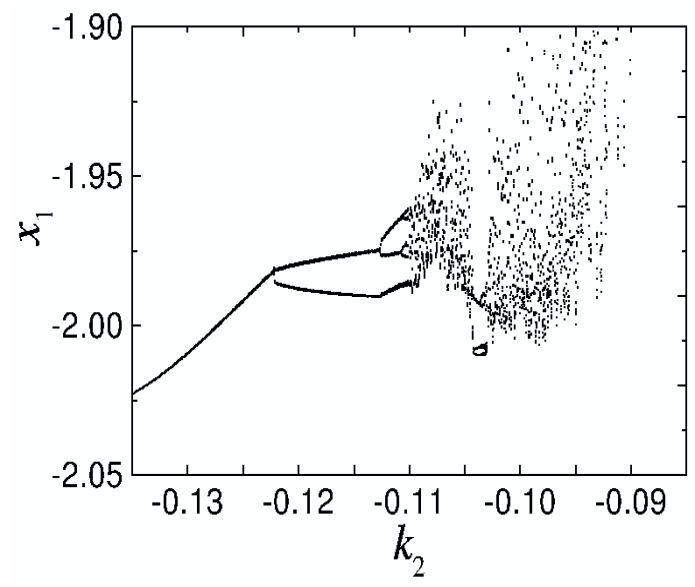

Fig. 2. Period doubling bifurcation diagram of controlled six-order coupled Chua's circuit with increasing $k_{2}$ (for $k_{3}=-0.2$ ) in Poincaré surface section $x_{2}=x_{5}=0$. 


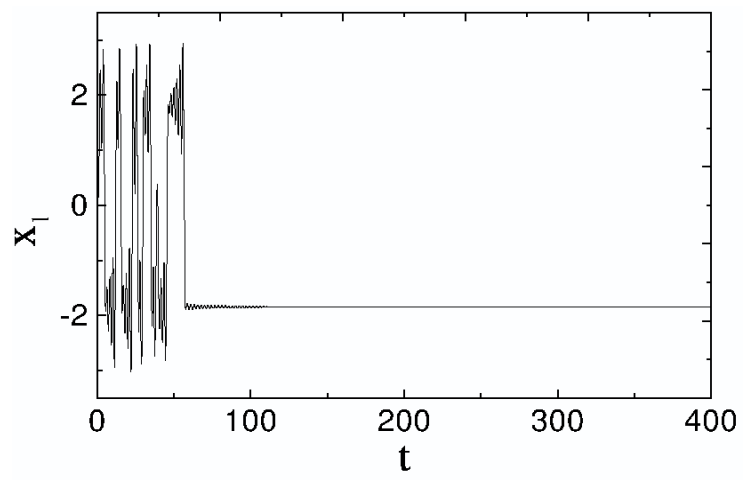

(a)

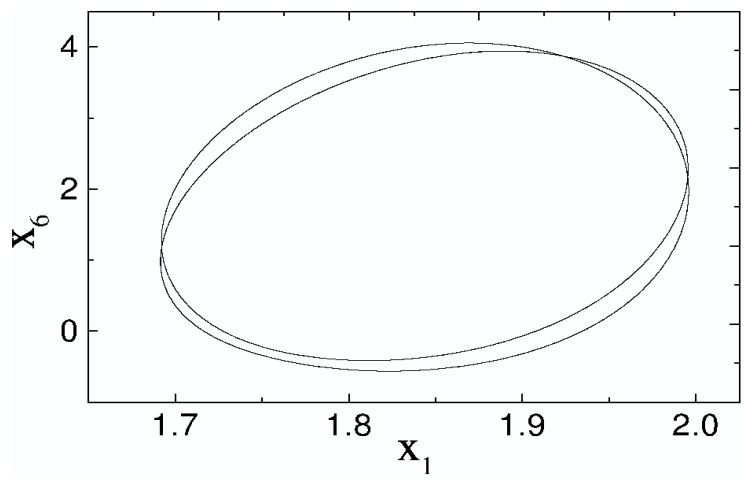

(b)

Fig. 3. (a) The controlling process approaching to fixed point $A_{1}$ for $k_{2}=-0.2, k_{3}=-0.3$. (b) The stabilized orbit of period-2 limit cycle with $k_{2}=-0.2, k_{3}=-0.12$.

To simplify the analysis, the control parameters $k_{1}$ is kept as 1.0 in the above theoretical discussion. Actually, chaos can still be controlled for $k_{1}<1$.0. However, the controlled system will approach to fixed points or periodic limit cycles not embedded in the original chaotic attractor. In other words, some new stable dynamical behaviors can be generated. Hence, using the method presented in this paper, not only the unstable periodic points embedded in original chaotic attractor can be stabilized, but also new stable dynamical behaviors may appear, that is, some new stable periodic orbits which are not existed in original system may be approached as attractors in controlled system.

This work is supported by the Natural Science Foundation of China (Nos. 10247005, 19932020, 19974039 and 70271070), the Special Funds for National Basic Research Major Project in China (973 Project), the China-Canada University Industry Partnership Program (CCUIPP-NSFC No. 70142005) and Guangxi Natural Science Foundation (No. 0135063). 


\section{References}

1. E. Ott, C. Grebogi and J. Yorke, Phys. Rev. Lett. 64, 1196 (1990).

2. B. Lima and M. Pettini, Phys. Rev. A41, 726 (1990).

3. Y. Braiman and I. Goldhirsch, Phys. Rev. Lett. 66, 2545 (1991).

4. K. Pyragas, Phys. Lett. A170, 421 (1992).

5. J. Guemez and M. A. Matias, Phys. Lett. A181, 29 (1993).

6. X. S. Luo, L. J. Kong and W. L. Qu, Acta Physica Sinica (in Chinese) 47, 1078 (1998).

7. G. Chen and T. Ueta, Int. J. Bifu. Chaos, 9, 1465 (1990).

8. G. Hu, Z. Qu and K. He, Int. J. Bifur. Chaos 5, 901 (1995).

9. L. O. Chua, M. Itoh, L. Kocarev and K. Eckert, J. of Circuits, System Computers 3, 93 (1993). 